\title{
Infrared and self-frequency-doubling emission characteristics of diode-pumped Nd:LGSB laser crystal
}

\author{
Catalina-Alice Brandus ${ }^{1}$, Madalin Greculeasa ${ }^{1,2}$, Alin Broasca ${ }^{1,2}$, Flavius Voicu ${ }^{1}$, Lucian Gheorghe ${ }^{1}$ \\ 1. National Institute for Laser, Plasma and Radiation Physics, Laboratory of Solid-State Quantum Electronics, Magurele 077125, Romania \\ 2. Doctoral School of Physics, University of Bucharest, Faculty of Physics, Magurele 077125, Ilfov, Romania
}

Visible laser sources are attractive for various applications, such as laser display, information storage, optical communications, or in medicine. In addition, a self-frequency-doubling (SFD) laser can be incorporated in a chip device, bringing advantages like stability, efficiency, low cost, compactness and simplicity. Different $\mathrm{Yb}^{3+}-$ and $\mathrm{Nd}^{3+}$-doped bifunctional crystals have been developed [1], including Yb:YCOB [2], Nd:GdCOB [3], Nd:NLBO [4], or Nd:CTGS and Nd:STGS [5,6]. The need for efficient SFD laser devices justifies the high interest for discovering novel bifunctional materials with enhanced performances [7]. In this work, we report on infrared laser emission and first SFD results at $531 \mathrm{~nm}$ achieved with a 2.3-at.\% Nd:LGSB bifunctional crystal, grown by the Czochralski method in our laboratory.

The laser medium was a 2.3-at.\% Nd:LGSB negative uniaxial crystal, uncoated, with a thickness of $5.7 \mathrm{~mm}$; it was cut for type I phase matching condition $\left(\theta=35.3^{\circ}, \varphi=60^{\circ}\right)$ at room temperature. The crystal was fixed in a copper mount whose temperature was carefully controlled, in order to ensure proper cooling during lasing, while keeping the phase matching condition for SFD. The optical resonator (Fig. 1) was a short (10-mm length) planeconcave configuration. The plane pump mirror (HRM) was coated high transmission (HT, T> 98\%) at the 807$\mathrm{nm}$ pump wavelength $\left(\lambda_{\mathrm{p}}\right)$ and high reflectivity $(\mathrm{HR}, \mathrm{R}>99.9 \%)$ at both fundamental $\left(\lambda_{\omega}=1062 \mathrm{~nm}\right)$ and green $\left(\lambda_{2 \omega}=531 \mathrm{~nm}\right)$ laser wavelengths. The concave out-coupling mirror $(\mathrm{OCM})$ had a radius $\rho=100 \mathrm{~mm}$. The pump (at $\lambda_{\mathrm{p}}=807 \mathrm{~nm}$ ) was done with a fiber-coupled diode laser, whose fiber end $(100-\mu \mathrm{m}$ core diameter, NA=0.22) was imaged into Nd:LGSB with a 1:1 optical system made of two aspherical lenses.

The laser emission at $\lambda_{\omega}=1062 \mathrm{~nm}$ was evaluated first, using OCM with different transmissions, T. The best performance was recorded for an OCM having $\mathrm{T}=0.05$. As shown in Fig. 2a), the Nd:LGSB crystal yielded 1.55 W output power $\left(\mathrm{P}_{\text {out }}\right)$ at $4.5-\mathrm{W}$ absorbed pump power $\left(\mathrm{P}_{\mathrm{abs}}\right)$; the slope efficiency, with respect to $\mathrm{P}_{\mathrm{abs}}$, was $\eta_{\mathrm{sa}}=$ 0.42. Saturation of $\mathrm{P}_{\text {out }}$ was observed for $\mathrm{P}_{\text {abs }}$ exceeding $3.9 \mathrm{~W}$, indicating thermal effects in Nd:LGSB.

For the generation of green light by SFD, an OCM coated HR (R>99.9\% at $1062 \mathrm{~nm})$ and HT (T> 95\% at $531 \mathrm{~nm}$ ) was used. The green output power reached $\mathrm{P}_{\text {out }}=13.2 \mathrm{~mW}$ for $\mathrm{P}_{\mathrm{abs}}=4.31 \mathrm{~W}$ at $807 \mathrm{~nm}$ [Fig. 2b)]. The spectrum was centred at $\lambda_{2 \omega}=531 \mathrm{~nm}$, having a bandwidth (FWHM definition) of $1.3 \mathrm{~nm}$.

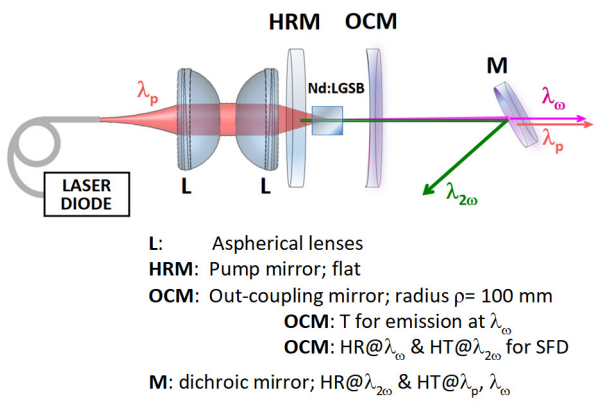

Fig. 1 The experimental set-up, general view.
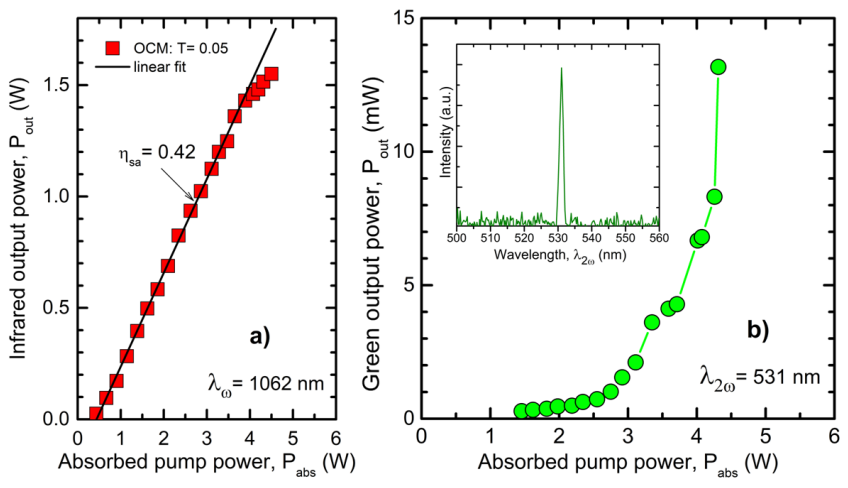

Fig. 2 Output power vs. aborbed pump power for emission at a) $\lambda_{\omega}=1062 \mathrm{~nm}$ and b) SFD at $\lambda_{2 \omega}=531 \mathrm{~nm}$. Inset shows the emission spectrum at $531 \mathrm{~nm}$.

In conclusion, we report on first SFD experiments performed on Nd:LGSB bifunctional laser crystal, from which $13.2 \mathrm{~mW}$ output power of green light at $531 \mathrm{~nm}$ was obtained. The results compare well with previous reports on other Nd-based bifunctional media [4-6]. Improved SFD performances are expected by optimizing the Nd:LGSB medium characteristics (doping level and length), by coating the crystal, as well as by designing a new laser resonator.

Financing: Projects 119/2017, PN-III-P4-ID-PCE-2016-0853 and NUCLEU 16N/2019, Ministry of Research and Innovation, Romania.

\section{References}

[1] A. Brenier, D. Jaque, A. Majchrowski, Opt. Mater. 28(4), 310-323 (2006).

[2] F. Khaled, P. Loiseau, G. Aka, and L. Gheorghe, Opt. Lett. 41(15), 3607-3610 (2016).

[3] H. Yu, N. Zong, Z. Pan, H. Zhang, J. Wang, Z. Wang, and Z. Xu, Opt. Lett. 36(19), 3852-3854 (2011).

[4] J. Zhang, H. Yu, Y. Li, L. Han, Y. Wu and H. Zhang, Opt. Lett. 37(17), 3501-3503 (2012).

[5] F. Chen, Y. Zhou, F. Yu, Y. Liu, S. Hou, H. Wang, Z. Wang, X. Duan, S. Guo, X. Zhao, J. Alloy. Compd. 651, 475-478 (2015).

[6] F. Chen, L. Wang, X. Wang, X. Cheng, F. Yu, Z. Wang, X. Zhao, Opt. Mater. 73, 33-37 (2017).

[7] L. Gheorghe, A. Broasca, M. Greculeasa, F. Voicu, G. Stanciu, S. Hau, G. Croitoru, C. A. Brandus, C. Gheorghe, F. Khaled, P. Loiseau, G. Aka, Opt. Mater. X 7, 100052 (2020). 